\title{
Los sujetos de la transición: notas sobre la dinámica de los actores y la transición democrática en Centroamérica ${ }^{1}$
}

\author{
Carlos Sojo \\ FLACSO-Programa Cosra Rica
}

San josé, 20 de febrero de 1994

\section{Resumen}

El objetivo del artículo es discurir algunos de los trabajos del grupo de investigación de la UAB, en el contexto de las transiciones democráticas en América Central. En primer lugar, se rechazan dos interpretaciones corrientes: "nada ha cambiado" y "el área está formada por democracias ya completas", y subraya algunas de las características básicas de aquellos procesos de cambio político. En segundo lugar, el autor discute algunos problemas metodológicos en la investigación sobre los actores políticos centroamericanos. Finalmente, se analizan las consecuencias de políticas socioeconomicas y de cambios en las relaciones civilmilitar.

Palabras clave: actores políticos, transiciones democráticas, políicas públicas, Centroamérica.

Abstract. The actors of transition: Notes about their dynamics and democratic transitions in Central America

The main goal of the article is to discuss some of the Papers produced by the UAB research group in the broader context of Central American democratic transizions. First, the article refuses two basic descriptions of Central American politics: "nothing has changed" or "there are full democracies in all the arean and highlights some of the basic characteristits of these processes of political change. Second, the article discusses some methodological problems in doing research about Central American political actors. Finally, the implications of socioeconomic policies and the changes in civic-military relations are analyzed.

Key words: polizical actors, democratic transitions, public policies, Central America.

\author{
Sumario \\ Introducción Los actores (sociales-políticos): tipologías \\ I.as coordenadas del cambio Los antagonismos dominantes: \\ bases de la prognosis
}

1. Ponencia presentada al panel tPolitical actors and political change in Cenrral Americas del XVIII International Congress of Latin American Studies Association, LASA. Atlanta, Georgia. 10-12 de marzo de 1994. 


\section{Introducción}

Los problemas de la región centroamericana a menudo han estimulado el trabajo de académicos fuera de la región, por razones muy diversas: en algunos casos por la causa tevolucionaria internacional que exigía la comprensión de la dinámica de las revoluciones sociales más alentadoras de la década de los ochenta; en otros casos por la magnitud y la peculiaridad de los enfrentamientos políticos que integraban en una sola matriz de contradicciones fuerzas armadas, gobiernos, oligarquías, movimientos revolucionarios, potencias y subpotencias extrarregionales; en algunos casos, dichosamente los menos, por la burda necrofilia del sensacionalismo periodístico.

Los centroamericanos, impulsados por el interés propio pero también obligados por el destino y las limitaciones financieras a permanecer atentos a la dinámica regional, vimos como con la paz, con el final del enfrentamiento entre oriente y occidente, y con la emergencia de una "frágil normalidad" en el plano económico y social, se fueron de la región muchos intereses, tanto académicos como políticos. Es por ello que resulta cstimulante conocer los esfuerzos actuales de colegas españoles del Grupo de Estudios Políticos Centroamericanos de la Universidad de Barcelona por derivar de la compleja coyuntura regional, elementos útiles para la comprensión de las nuevas dimensiones de la dinámica social y política que caracteriza la transición democrática en Centroamérica. Los analistas de la UAB han sabido aprovechar la distancia como una ventaja comparativa que les permite sustraerse de lo meramente casuístico, anecdótico o circunstancial y colocarse en el plano de la explicación reórica, si bien todavía embrionaria, ciertamente necesaria para la comprensión de los alcances del cambio político experimentado y sus debilidades y fortalezas más visibles.

A continuación presento algunas reflexiones surgidas a raíz de la lectura de varios trabajos sobre la dinámica de los actores políticos centroamericanos, en particular para los casos de Nicaragua y Guatemala ${ }^{2}$. No se trata de referencias específicas o críticas particulares, sino más bien de ideas en torno a los tres elementos que a mi juicio están presentes como variables relevantes en los análisis indicados: se trata en primer lugar de la cuestión de las coordinadas del cambio ${ }^{3}$, es decir, de la identificación del entorno sociopolítico y económico

2. Los trabajos leidos, que motivan las reflexiones siguientes son: FABRFCiAs, Joan Font. Hacia un mapa de los actores poltticos guatemaltecos. FONT, Joan; GOMA, Ricard. Actores y cambio poltico en Centroamérica: un marco de analisis. GoMA, Ricard. De la revolución al neoliberalismo: actores, estrategias y politicas públicas. Ponencias presentadas a las jornadas de debate "Quinze anys de canvi polític a Centreamèrica". Universitat Autonoma de Barcelona. Enero de I994. También de FoNT y GokA: Cambio y actores politicos en Centroamerica: Los casos guatemalteco y nicaragiense. Ponencia presentada al III Encuencro América Latina Hoy, Madrid, 1991. aEl proceso de democratización en Nicaragua: actores, estrategias y confliceo." En Afers Internacionals, núm. 20, p. 49-75.

3. Las enriendo como la combinación de las dimensiones de la vida social y política en las que se observa el cambio, con la identificación de una situación de partida contrastada con la situación actual. Por ejemplo, el cambio policico-institucional riene puntos de partida en la guerra civil y una situación actual caractcrizada por la negociación y los acuerdos de paz. 
que tipifica a la situación actual de los páses de Centroamérica como "transición". En segundo lugar, de la cuestión relativa a la identificación de actores políticos y finalmente el señalamiento de los principales antagonismos que se presentan en la actualidad.

\section{Las coordenadas del cambio}

Aunque sea difícil de precisar, algo cambió en Centroamérica si comparamos la situación de 1994 con, digamos, un lustro atrás. Hace cinco años la región estaba sumida en una violenta guerra atizada por la intervención de potencias extranjeras que apoyaban gobierno o insurgentes. La gravedad de la crisis, que amenazaba con prolongarse hasta trascender los límites de la guerra civil, había disminuido como resultado de los esfuerzos negociadores emprendidos por los presidentes centroamericanos a partir de mayo de 1986 con la reunión de Esquipulas. En el escenario político, la sociedad civil de Centroamérica se dividía en el enfrentamiento ideológico de dos modelos de desarrollo: uno autodenominado popular y otro autodenominado democrático ${ }^{4}$. En el plano económico, la inestabilidad en mucho asociada a la guerra, producía el deterioro constante de indicadores macroeconómicos y condiciones sociales de vida. Tal deterioro se agravaba por la aplicación, en condiciones políticas adversas, de propuestas de política económica que no ponían atención más que a los equilibrios monetarios, fiscales y comerciales ${ }^{5}$.

Para establecer el cambio de tal situación con respecto al momento actual, estamos obligados a reconocer y diferenciar las zonas de la actividad política y social en donde ocurren tales modificaciones. De lo contrario corremos el riesgo de sobrestimar el cambio (por ejemplo en las visiones minimalistas según las cuáles lo democrático se limita a la celebración periódica de comicios justos), o subestimarlo (si se enfatiza la ausencia de cambios estructurales en las relaciones cívico-militares o en la política económica).

Examinando las tres dimensiones en las que pueden situarse experiencias de cambio, esto es la forma de elección de gobernantes, la cuestión cívico-militar y la cuestión económico-social, proponemos la siguiente interpretación:

a) Modernización de la mecánica electoral: A esta altura, parece bastante claro que el único instrumento legítimo para la elección de gobernantes está basado en la consulta popular periódica. El cambio es importante porque, con la excepción de Guatemala, no parece haber duda entorno a la imposibilidad del retorno a situaciones de hecho para definir gobiernos. Exceptuar a Guatemala parece injusto si se considera el desenlace de la crisis inicia-

4. Las autodenominaciones muestran a menudo fuerces discrepancias con la realidad.

5. En Nicaragua, El Salvador y, por supuesto, Guatemala las reformas económicas de orientación neoliberal comenzaron a aplicarse (con presencia de los organismos multilaterales de financiamiento o sin clla) antes del logro de acuerdos de solución negociada a los conflictos politico-militares. 
da en mayo de 1993 por el autogolpe del presidente Serrano, pero no lo es tanto si se toma en cuenta la "apatía" con que los ciudadanos registrados se enfrentan a las consultas populares, como quedó de manifiesto en el reciente referéndum sobre reformas constitucionales.

Por otra parte hablamos de modernización de la mecánica electoral, porque es evidente que se ha avanzado en la técnica aunque no mucho en la cultura cívica. Las fuerzas políticas están aprendiendo el uso de instrumentos de tecnología electoral, y los gobiernos están interesados en la construcción de "instituciones democráticas", pero, con probables excepciones, no están muy ocupados en activar mecanismos de consulta popular permanente o de ampliación de la participación ciudadana (v.g. descentralización y poder local). Los partidos, en general, funcionan como maquinarias de transporte de votantes, pero escasamente como instancias de representación o intermediación legítima de intereses sociales. Las «instituciones democráticas" pueden contribuir a establecer y mantener vigentes reglas de juego (equitativas y justas) político, pero sus márgenes de maniobra son todavía limitados en virtud de la presencia activa de instituciones y prácticas más bien orientadas al pasado autoritario. Aunque en Guatemala el desencanto por la política es dramático, en los demás países es común observarlo en la forma de crítica a los «políticos tradicionales".

b) Desgaste del enfrentamiento politico-militar. De las tres guerras civiles que caracterizaban la situación centroamericana cinco años atrás, sólo queda una. No obstante, en Nicaragua, la frustración económica - muy frecuente entre ex combatientes de uno y otro bando-, más que cualquier otra contradicción, ha mantenido encendidas algunas mechas. En El Salvador las sombras de la guerra sucia se resisten a desaparecer.

Los avances observados, sin embargo, no autorizan a afirmar que hay un cambio sustantivo en la relación cívico-militar. Los ejércitos se han redimensionado pero todavía pesan más de lo debido en términos políticos y económicos. Las dramáticas disminuciones de efectivos (del orden del $50 \%$ en el Salvador y del $83 \%$ en Nicaragua) no se reflejan proporcionalmente en reducciones de gasto militar. En Honduras, la retórica de la reconversión militar ${ }^{6}$, autoriza a los dirigentes castrenses a mantener niveles sobredimensionados de gasto, justificados a veces por absurdas iniciativas como la llamada ubatallones verdesn: soldados que para mantenerse activos realizan campaña de siembra de arbolitos. Como resultado, en Centroamérica no hay "dividendos de la paz».

6. En los países en donde ha disminuido o cesado la presencia del «enemigo internou los militares, a fin de mantener sus niveles de gasto y sus correlativas cuotas de poder político, están muy interesados en las llamadas "nuevas amenazas a la seguridad" como el narcotráfico, la depredación ambiental, la contaminación, erc. No toman en cuenta, sin embargo, que los analistas se refieren, a menudo, a éstas como amenazas "no militares", lo que indica que no son los ejercitos los mejor capacitados para enfrentar tales desafíos. A esta parte del análisis, los generales ponen oídos sordos. 
Tampoco hay toletancia militar a una mayor intervención de los civiles en la definición y el control de las políticas de seguridad y defensa, como corresponde a sociedades democráticas. En la mayoría de los países la sujeción al poder civil es condicional, es decir, depende del consentimiento gubernamental a la autonomia de las fuerzas armadas. Tal consentimiento, por ejemplo, impide u obstaculiza la aplicación de la justicia civil al estamento militar, lo que garantiza entre otros vicios antidemocráticos la persistencia de la impunidad.

c) La estrategia de desarrollo común en todos los países está basada en un relanzamiento de las economías al mercado internacional, por medio de la promoción de exportaciones, la apertura comercial y la disminución del tamaño del Estado y de sus funciones económicas. Un corolario de este proceso es la activación de mecanismos de compensación social orientados, preferentemente (aunque no exclusivamente) a los grupos de más bajo ingreso afectados por las políticas de ajuste económico.

En los países de enfrentamiento militar disminuido, la cuestión social ha ocupado un lugar de preferencia en el debate político. Este debate se da dentro de dos tipos de lindero: los del reconocimiento a la necesidad de la compensación económica (más como criterio de estabilización política que como componente integral del plan de desarrollo económico) y la convicción de que no hay propuesta alternativa viable a la política económica dominante. En Costa Rica y en Honduras la cuestión planteada en las recientes elecciones fue la de flexibilizar el ajuste y otorgar a la política gubernamental un mayor contenido social. En El Salvador, los acuerdos de paz de Chapultepec y el desarrollo posterior de las conversaciones en el seno del Foro de Concertación Económico-Social han partido de la afirmación (aceptada por las fuerzas de izquierda) de que la estrategia economica de ARENA (el "modelo") no estaba sujeto a negociación.

En Guatemala, donde la persistencia del enfrentamiento militar y de las prácticas políticas autoritarias mantienen "la política" en el centro de las preocupaciones de numerosos grupos gremiales, es notorio que parte de las dificultades que ha experimentado el Gobierno de Ramiro de León está en la doble herida provocada por cambios innecesarios en la política de negociación con la URNG y la ausencia de cambios necesarios en la conducción de la política económica y social.

En esta dimensión de la vida centroamericana, las coordenadas del cambio pasarían por una progresiva generalización de las políticas de ajuste estructural, al tiempo que empieza a tomar forma una preocupación "reactiva" por los problemas de la distribución, pero no en sentido universal y de largo plazo, sino es sentido foralista y de corto plazo.

La suma de estos cambios y continuidades arroja un producto ciertamente diferente, un nuevo entorno dentro del cual se mueven y al cual configuran las fuerzas políticas y sociales centroamericanas. No hay un cambio unívoco; 
el que existe es uno multidimensional, más profundo en unos campos, menos en otros. Lo que es importante anotar a continuación es que el comportamiento y la configuración de tales fuerzas es distinto según sea la dimensión en que operan. Nuestra opinión es que no existen fuerzas políticas o sociales constituidas de previo, al margen de los hechos sociales que constituyen y a la vez las conforman. De otra forma se corre el riesgo de suponer que las circunstancias históricas se modifican al calor de la acción de fuerzas políticas y sociales, mientras éstas permanecen inalteradas.

\section{Los actores (sociales-políticos): tipologías}

El riesgo de las tipologías es el de la síntesis y la omisión: ¿están todos los que son?, ¿son todos los que están? Sus ventajas para el desarrollo del conocimiento son indiscutibles, de lo contrario es prácticamente imposible reconstruir comportamientos sociales y políticos que aparecen como una suma abigarrada y confusa de opiniones personales, protestas anónimas, egos enardecidos.

Existen sin embargo multitud de opciones metodológicas, una de las cuales nos resulta particularmente problemática: esta es la definición de fuerzas o agrupamientos según sea su espacio de incidencia político o social. La pregunta es ipueden aislarse sin graves consecuencias fuerzas políticas de fuerzas sociales? Sin duda que ello puede hacerse si se considera que las unas se constituyen predominantemente alrededor de antagonismos de poder, mientras que las otras se relacionan conflictivamente en el campo de la producción.

Siendo así se podría entonces considerar que la lucha política ocurre dentro del Estado, mientras que la lucha social se desarrolla en el seno de la sociedad civil. La lucha de clases, como es sabido, tiene lugar en el conjunto del bloque histórico. Pero ¿qué pasa cuando los antagonismos emergentes no pueden situarse en un solo piso y, simultáneamente, las fuerzas sociales y políticas actuantes son estructuralmente heterogéneas?

Por ejemplo, los acuerdos de paz en El Salvador son claramente el resultado de una negociación en el seno del Estado entre fuerzas políticas que defienden modelos antagónicos de sociedad. Como el resultado no es revolucionario (suma cero), la manifestación de este acuerdo político no se circunscribe al cambio desde arriba, sino que demanda modificaciones en la estructura social de una magnitud tal que, no obstante, sean compatibles con el modelo económico y político vigente. La cuestión es la de integrar política y económicamente la sociedad, introduciendo los excluidos y no al revés.

Por otro lado está el hecho de que las demandas políticas, por ejemplo el respeto a los derechos humanos, la libre participación política o la disminución de la influencia de las fuerzas armadas, no pueden situarse de manera exclusiva en el prontuario de ciertas clases sociales. Se trata de luchas políticas que «constituyen» (Laclau y Mouffe) nuevos sujetos sociales de heterogénea composicion estructural.

Lo mismo ocurre con demandas económico-sociales. Ante la apertura indiscriminada al mercado internacional es difícil diferenciar las protestas de peque- 
ños productores familiares (campesinos o artesanales) de las de los grandes industriales o productores agrícolas protegidos. La política de privatización, por otro lado, "divide» el campo de lo popular al confrontar usuarios-consumidores, empleados públicos o productores. La eliminación de regulaciones públicas (de precios) o subsidios o la eliminación de barreras a la importación tiene un efecto semejante entre consumidores y productores. La inflación favorece a los productores en el corto plazo y puede ocurrir, aunque no siempre pasa, que la apertura de mercados, por ejemplo de granos, abarate el costo de la canasta básica familiar.

Por todo lo anterior, nos parece que una adecuada identificación de la acción de fuerzas políticas y sociales en la transición de Centroamérica a la democracia, puede partir de la identificación de posiciones políticas (partidos y fuerzas militares), corporativas y estructurales, étnicas y culturales, basada en la propia autodefinición de estos grupos, pero no debe quedarse ahí. La identificación de las posiciones subjetivas debe conducir al señalamiento de nudos problemáticos, de áreas de antagonismo, a partir de las cuales las diversas agrupaciones se reorganizan constituyendo nuevas fuerzas sociales. Los antagonismos particulares, y no los agrupamientos específicos (es decir las fuerzas), sí pueden identificarse como políticos (si se refieren al poder) o sociales (si se refieren a la producción-distribución).

\section{Los antagonismos dominantes: bases de la prognosis}

La definición adecuada de las fuerzas sociales y políticas que actúan en la región y de las posibilidades de su evolución futura está estrechamente asociada a la observación de los principales antagonismos que confrontan. Las razones ideológicas y culturales pueden ser de muy diverso origen, pero los antagonismos básicos se presentan como diferencias en torno a cuestiones particulares. Según mi entender, los principales antagonismos que organizan las posiciones actuales de las fuerzas políticas y sociales de Centroamérica están asociados a: 1) las implicaciones políticas de las reformas económicas y 2) las implicaciones sociales de la situación político-militar.

\section{Implicaciones politicas de las reformas económicas}

Uno de los principales aspectos económicos del ajuste, que tiene particular proyección política para el caso centroamericano, se refiere a la reforma del Estado. El objetivo primordial de los reformadores neoliberales es la disminución al mínimo posible de las actividades del Estado y de su intervención en la economía. Esta reforma, sin embargo, resulta conflictiva en una región que no ha logrado construir un aparato de Estado capaz, en el mínimo, de garantizar estabilidad social, bienestar y crecimiento económico. En casos críticos como el nicaragüense el propósito de la reforma debería ser más bien la ampliación de las actividades públicas, particularmente en gestión económica y distribución social (o bienestar). En otras palabras, en Nicaragua no existe el 
Estado mínimo necesario para hacer frente a las exigencias de gobernabilidad de la sociedad. En otros casos, en donde existen mayores espacios de acuerdo político, está claro que la expectativa de gobernabilidad requiere un nivel de acción del Estado, que si bien debe ser sostenible desde el punto de vista fiscal, se aleja mucho de la utopía reduccionista de los defensores ciegos del equilibrio neoliberal. Así to demuestran los frenos políticos a la disminución del Estado en Costa Rica, o la ampliación de actividades, especialmente en el plano de la distribución social, del Estado salvadoreño en el marco del Plan de Reconstrucción Nacional de la posguerra.

Pero, en general, se observa que las principales consecuencias sociales de tales reformas están asociadas a la disminución de los recursos financieros del Estado dirigidos a mitigat los efectos sociales de la apertura económica. En cl escenario actual de control del gasto público, generalmente son las actividades sociales y redistriburivas las primeras afectadas por recortes presupuestarios, considerando que el Gobierno debe satisfacer, por una parte, las necesidades de financiamiento a las actividades productivas y, por otra, las expectativas de grupos burocráticos que se resisten a disminuir sus regalías. Esto es particularmente serio en lo que se refiere al control y la disminución de los gastos de defensa.

Además, la relativa estabilización política del área, junto a la aparición de nuevos focos de conflicto internacional o de atracción del interés de la cooperación internacional (en particular la canalización de fondos de cooperación internacional de Occidente hacia las emergentes democracias de Europa det Este y la antigıa Unión Soviética) han disminuido la disposición de recursos financieros concesionales por parte de los países de la región. Más aún, Estados Unidos, que invirtió cerca de 8.000 millones de dólares en ayuda económica no reembolsabie a Centroamérica durante la década de los ochenta, ha empezado a reducir, en algunos casos como Costa Rica totalmente, su ayuda a los paises del área. En compensación se ofrece un nuevo trato comercial y mayores inversiones que todavía no parecen capaces de variar significativamente en relación con las prácticas de la década anterior.

En lo concerniente al comercio está claro que una parte importante de las actividades productivas beneficiadas con acuerdos de acceso preferencial al mercado norteamericano (en el marco de la iniciativa para la Cuenca del Caribe) se verían afectadas negativamente con la eliminación de trabas para el acceso de productos originarios de países que pueden desarrollar mayores economías de escala y que tienen prioridad en las negociaciones comerciales de los Estados Unidos: primero México, después Chile y quizá otros paises grandes de América del Sur. En lo relativo a las inversiones, la práctica común ha sido que la mayoría de las inversiones extranjeras a Centroamérica se ha colocado en el sector financiero panameño y no parecen existir incentivos suficientes como para trasladar buena parte de ellas hacia los sectores productivos de los demás países. Por ello, en términos generales, la finalización de la ayuda concesional a Centroamérica no ha encontrado todavía un sustituto positivo para los intereses de la región. 
Lo anterior es importante porque es difícil imaginar una reforma económica con estabilidad social en ausencia de compensación financiera por parte de la comunidad internacional. Costa Rica, país que inició más temprano que los demás la reforma estructural de su economía y que la ha desarrollado dentro del ambiente tradicional de estabilidad social y política que lo caracteriza, ha hecho las reformas más severas desde el punto de vista social en un periodo (1986-1990) en el que todavía disponía de la subvención financiera de los Estados Unidos. Es difícil esperar que con menores subvenciones y más hostilidad en el ambiente comercial internacional otros países del área, que carecen de la red de seguridad social construida a los largo de cuarenta años en Costa Rica, puedan hacer frente con sus propios recursos a los efectos sociales de la reforma económica. Al menos no en un ambiente de tranquilidad social.

\section{La cuestion politico-militar}

Una región que hasta hace poco tiempo se encontraba sumida en la guerra no resuelve de la noche a la mañana la problemática cuestión de las relaciones cívico-militares. Las implicaciones sociales de esta problemática, en términos de confrontación de fuerzas, tienen relación directa con las dimensiones políticas y sociales de la relación.

Por lo que toca a las dimensiones políticas, está lamentablemente claro que todavía persisten en la región sectores interesados en el tuso de la fuerza como única vía de satisfacción de intereses. El uso de la fuerza es todavía un arma de presión política que erosiona, en primer lugar, el disfrute de libertades y derechos básicos (como la libertad de conciencia y el derecho a la vida), y que, en segundo lugar, disminuye la legitimidad del Estado como único ejecutor de funciones represivas. A ello contribuye la autonomía relativa de las instituciones castrenses respecto del poder civil que puede ser interptetada como "privatización" de las funciones de represión y ejercicio legitimo de la fuerza.

Las fuerzas sociałes y polfticas de la región circulan alrededor de las posiciones de los grupos militares (estatales o insurreccionales), lo que manifiesta una sobredimensionada presencia de la cuestión político-militar en el debate nacional. En Nicaragua la ausencia de proyectos nacionales de desarrollo entre las fuerzas políticas de derecha coloca la cuestión del EPS en primer lugar de la agenda. La imagen se repite con expresiones distintas pero connotaciones semejantes en los demás países del área con la evidente excepción de Costa Rica.

I as dimensiones sociales se refieren a los frenos que la presencia de inadecuadas relaciones cívico-militares colocan en las posibilidades de desarrollo económico y social de los países del área.

En primer lugar el tema ya mencionado de los masivos gastos militares que no parecen modificarse corno efecto de las reducciones de efectivos, y que tampoco parece probable que estén sujetos en el futuro al escrutinio popular por medio de la discusión legislativa de los gastos y la política de defensa nacional. 
Como efecto de este masivo gasto, los Estados centroamericanos no disponen de los recursos fiscales necesarios para hacer frente a las tareas de la reconstrucción económica y social.

Además se observa el efecto de lo que denominamos la especialización perversa de la fuerza de trabajo. Arrojados a las armas por circunstancias que no les eran claras, pero cuyos efectos no les eran ajenos, decenas de miles de mujeres y hombres del campo, de las ciudades, estudiantes y profesionales, dejaron sus respectivas actividades cotidianas y productivas. Hoy día, guerrear es lo único que saben hacer. Las declaraciones de los recontras, recompas o revueltos en Nicaragua y la ola de asaltos en El Salvador son la manifestación de nuevas formas de delincuencia que no aparecen asociadas a las sociopatías tradicionales, sino a los desequilibrios provocados por sistemas económicos y sociales frustrantes en el pasado y en el presente. Las reivindicaciones de los secuestradores nicaragüenses son una combinación de consignas políticas -a menudo incoherentes o imposibles - (que buscan un efecto legitimatorio, que confirma la tesis de que la cultura política tolera y legitima el uso de la fuerza como mecanismo de presión política) con demandas económicas que parecieran ser la motivación central.

Lo que parece común a las dirmensiones políticas y económicas de la crisis en las relaciones cívico-militares de Centroamérica es que en buena medida impiden el levantamiento de cuestiones sociales relevantes para la reconstrucción de largo plazo. La manifestación más dramática de la transición política en Centroamérica es que lo autoritario se resiste a morir y a dejar nacer nuevas formas de intermediación política. 